\title{
A SYSTEM FOR QUANTIFYING BEHAVIOR OF NEONATE CATERPILLARS AND OTHER SMALL, SLOW-MOVING ANIMALS
}

\author{
Sanford D. Eigenbrode, John Barnard ${ }^{1}$, and Anthony M. Shelton \\ Department of Entomology, New York State Agricultural Experiment Station, Cornell University, Geneva, \\ New York, USA 14456
}

We have developed a system for recording and quantifying animal behavior on artificial or natural substrates. The system is designed for subjects such as insects and mites with movement rates as high as $4 \mathrm{~cm} / \mathrm{min}$. The principle is the same as in automatic stage or sphere centering devices (Berg 1971; Kramer 1976; Thiery and Visser 1986), but the compensations are made manually by an observer who may also simultaneously enter codes for specific behaviors on a microcomputer keypad. Data files produced are analyzed or plotted using programs in SAS ${ }^{\circledR}$ (SAS Institute 1985a) or similar data analysis packages.

The equipment required is any Apple ${ }^{\circledR}$ Macintosh $\left.{ }^{(\boxplus i}\right)$ computer, a Summagraphics $^{\circledR}$ SummaSketch ${ }^{\circledR}$ tablet, a boom-mounted dissecting microscope equipped with an ocular crosshair, and a mechanical stage manipulator salvaged from a compound microscope. The "mouse" of the tablet is rigidly attached to a movable plastic stage operated by the stage manipulator. The stage and attached mouse move on the tablet, over which the dissecting microscope is mounted. During operation, the user manipulates the stage mechanism to maintain the image of the subject in the ocular crosshair of the microscope while entering single letter codes for specific behaviors on the computer keyboard. Artificial or natural substrates, such as host leaves, may be attached to the stage.

The tracking program is a compiled application created in Microsoft ${ }^{\circledR}$ QuickBASIC. The application uses the Macintosh operating system to interface with the operator through dialogue boxes. The program prompts the user for file name, maximum observation time for an individual, treatment information, coordinates of the test arena, and movement sensitivity (limited by hardware to $\geqslant 0.00254 \mathrm{~cm}$ ). The output data file contains the header information, a series of $X$ and $Y$ coordinates for the subject's position, elapsed time, and behavior codes. Output data files are subsequently condensed by a SAS program to provide total elapsed time, total distance covered, rate of movement, net turning angle (in which left and right turns have different signs), gross turning angle, net and gross turning rates per unit distance and unit time, total number of events for each of the user-specified behaviors, and total amount of time spent in each behavior for each individual. Other summary statistics can be produced with minor changes. Statistical comparisons can then be made using standard SAS procedures. Additional programs in SAS/Graph ${ }^{\circledR}$ (SAS Institute 1985b) create a plot of each animal's movement track and an ethogram in which the behaviors are plotted versus time.

We have used this system for analysis of the behavior of neonate diamondback moth larvae, Plutella xylostella (L.), on plant wax substrates. The insects are tiny $(<1 \mathrm{~mm})$ and move at approximately $1 \mathrm{~cm} / \mathrm{min}$. The behaviors of interest are walking, biting, spinning silk, tasting, and searching. Figure 1 presents a sample larval track (A) and ethogram (B) from an individual (observed for $5 \mathrm{~min}$ ).

The system is ideal for simultaneously quantifying observations of specific animal behaviors and movement. For studies in which only movement must be analyzed, fully automated recording systems, such as those cited above, in conjunction with analysis programs such as "TRAK" (developed and distributed by W.J. Bell, Kansas University, Lawrence, KS, USA 66045) may be more suitable. With mechanical or optical modifications, or both, our system is adaptable for observing smaller or larger subjects. Our system's advantages include: (1) required equipment is either low-cost or readily available in research laboratories; (2) behaviors and movements are recorded simultaneously; (3)

'Computer Services, New York State Agricultural Experiment Station, Cornell University, Geneva, New York, USA 14456. 
A

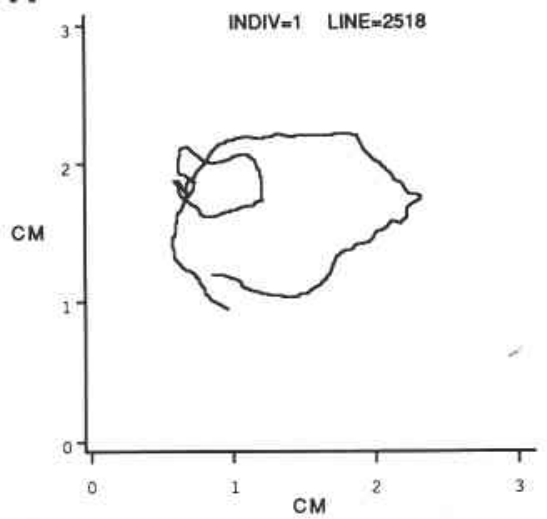

B

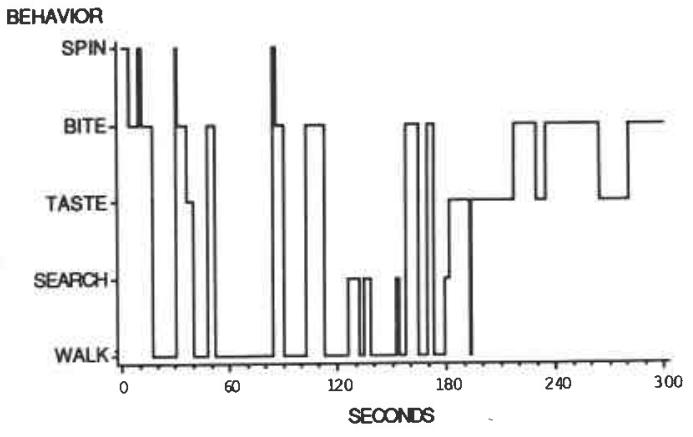

FIG. 1. Graphics output: (A) movement track for a single diamondback larvae recorded for 5 min; (B) ethogram of the same individual. (Plots in A and B were produced by SAS using a Tektronix 4014 emulator on the Macintosh.)

data files are produced that may be manipulated by SAS or other statistical packages; (4) behaviors may be monitored on natural substrates such as leaf surfaces.

Tracking application and SAS programs are available from the authors on request.

We thank R.E. Charlton for reviewing the manuscript.

Berg, H.C. 1971. How to track bacteria. Rev. Sci. Instrum. 42: 868-871.

Kramer, E. 1976. The orientation of walking honeybees in odour fields with small concentration gradients. Physiol. Ent. 1: 27-37.

SAS Institute. 1985a. SAS User's Guide: Basics. SAS Institute, Cary, NC. 1985b. SAS/Graph ${ }^{\circledR}$ User's Guide. SAS Institute, Cary, NC.

Thiery, D., and J.H. Visser. 1986. Masking of host plant odour in the olfactory orientation of the Colorado potato beetle. Entomologia Exp. Appl. 41: 165-172.

(Date received: 15 May 1989; date accepted: 30 August 1989) 